\title{
The libration and interior structure of large icy satellites and Mercury
}

\author{
Tim Van Hoolst ${ }^{1,2}$ \\ ${ }^{1}$ Royal Observatory of Belgium \\ Ringlaan 3, B-1180 Brussels, Belgium \\ email: tim.vanhoolst@oma.be \\ ${ }^{2}$ Institute of Astronomy, KU Leuven \\ Celestijnenlaan 200D, B-3001 Leuven, Belgium
}

\begin{abstract}
Longitudinal librations are periodic changes in the rotation angle of a planet or satellite. Their observation and subsequent interpretation have profoundly increased our understanding of the interior structure of Mercury. Likewise, libration is thought to provide important constraints on the interior structure of icy satellites. Here we study the libration of Mercury and large icy satellites rotating synchronously with their orbital motion and explain how it depends on the interior structure.
\end{abstract}

Keywords. planets and satellites: individual (Mercury), geodesy, rotation, icy satellites

\section{Introduction}

Most large and medium-sized (radius larger than $200 \mathrm{~km}$ ) satellites of the solar system are in a synchronous spin-orbit resonance in which their rotation period is equal to their orbital period. The situation arises as a result of dissipation in the satellite associated with tides raised by the central planet. In this stable state of synchronous rotation the longest axis of the satellite points towards the central planet at pericenter. Mercury is in a 3:2 spin-orbit resonance as has been postulated for the first time by Giuseppe Colombo based on observations of Pettengill and Dyce in 1965. Before that time, it was thought that Mercury's spin was in a 1:1 resonance with its orbital motion, just like the Moon and other satellites in their orbit around their central planet. The resonant rotation of Mercury is stable like the 1:1 resonance of the rotation of the satellites.

Stability, however, does not imply that the rotation rate is constant. Because of the difference in rotation period and orbit period, the long axis of Mercury rotates with respect to the direction to the sun. Also the long axis of satellites is generally not oriented in the direction to the central planet due to the eccentricity of the satellite orbits and the resulting variable orbital speed. The central body (the sun or the central planet) therefore exerts a time-variable gravitational torque on Mercury and on synchronously orbiting satellites. Consequently, Mercury and the satellites vary their rotation rate about the mean rate and the direction of the long axis librates with respect to its orientation at constant mean rotation rate (forced longitudinal libration).

Here we focus on the libration at the orbital period of Mercury (often referred to as the annual libration) and of the satellites (mostly referred to as the diurnal libration). We describe the expected libration behavior and show that observations of librations provide information on the interior structure. For example, a global liquid layer such as a subsurface ocean strongly affects the rotational dynamics and leads to a transfer of angular momentum between solid and liquid layers. Rotation observations have already been used to prove that Mercury's core is at least partially liquid and can demonstrate 
the existence of an internal ocean in icy satellites. Librations can also be used to further characterize different layers. As a prime example of this, the size and mean density of Mercury's core have recently been well constrained by libration observations (Hauck et al. 2013, Rivoldini \& Van Hoolst 2013).

\section{Mercury}

From the determination of the epoch and time delay that maximize the cross correlation of back-scattered radar signals at two antennas separated by a long baseline, Margot et al. (2007, 2012) have estimated the annual libration amplitude of Mercury to be $(38.5 \pm 1.6)$ arcsec, which corresponds to a maximum displacement at the equator with respect to the equilibrium rotation of $(455 \pm 19) \mathrm{m}$. In 1976 Peale had already realized that the observation of the annual libration of Mercury at about 88 days (the period of revolution of Mercury around the sun) is central to improve our understanding of the interior of Mercury. In particular it allows estimating the sizes of the core and the silicate shell (mantle plus crust). The reason for this lies in how Mercury reacts to the gravitational forcing of the sun. This can most easily be described by the equation for the conservation of angular momentum.

We will neglect in this analysis Mercury's small obliquity (the observed value is $(2.04 \pm$ 0.08) arcmin, Margot et al. 2012) and assume principal axis rotation at a rate $\Omega$ in this study of Mercury's rotation rate. Only the polar or z-component of the angular momentum must then be considered. For an entirely solid planet, we have

$$
C \dot{\Omega}=L
$$

where

$$
L=\frac{3}{2}(B-A) \frac{G M_{\odot}}{r^{3}} \sin 2 \xi
$$

is the solar torque on Mercury's permanent figure. We neglect the tidal torques as their effect is well below the observational precision (Van Hoolst et al. 2012). The distance $r$ is the distance between the mass centers of the sun and Mercury, and $\xi$ is the angle between the direction of Mercury's axis of smallest moment of inertia $A$ (also called the long axis) and the direction to the sun:

$$
\xi=f-\phi
$$

where $f$ is the true anomaly and $\phi$ the rotation angle between the long axis of Mercury and the major axis of Mercury's orbit considered fixed. The torque is seen to depend on the orbital and rotational motion of Mercury through its dependence on the angle $\xi$ and the distance $r$. It also depends on the equatorial shape of Mercury as given by the equatorial moment of inertia difference $B-A$. An accurate value for this quantity has only recently become available by the determination, by means of radio tracking the MESSENGER spacecraft, of the Hermean gravity field, in particular the degree-two and order-two coefficient $C_{22}=(B-A) /\left(4 M R^{2}\right)=(0.809 \pm 0.01) \times 10^{-5}$ (Smith et al. 2012). Prior to this accurate determination, interpretations of observed librations were seriously hampered (e.g. Margot et al. 2007).

Since the rotation is close to the $3: 2$ spin-orbit resonance, we introduce the small libration angle $\gamma=\phi-\frac{3}{2} M$, where $M$ is the mean anomaly. By rewriting the torque in terms of the libration angle and the mean and true anomaly and expanding the timedependent factor $\sin (2 \gamma+3 M-2 f) / r^{3}$ of the torque in terms of the mean anomaly, we 


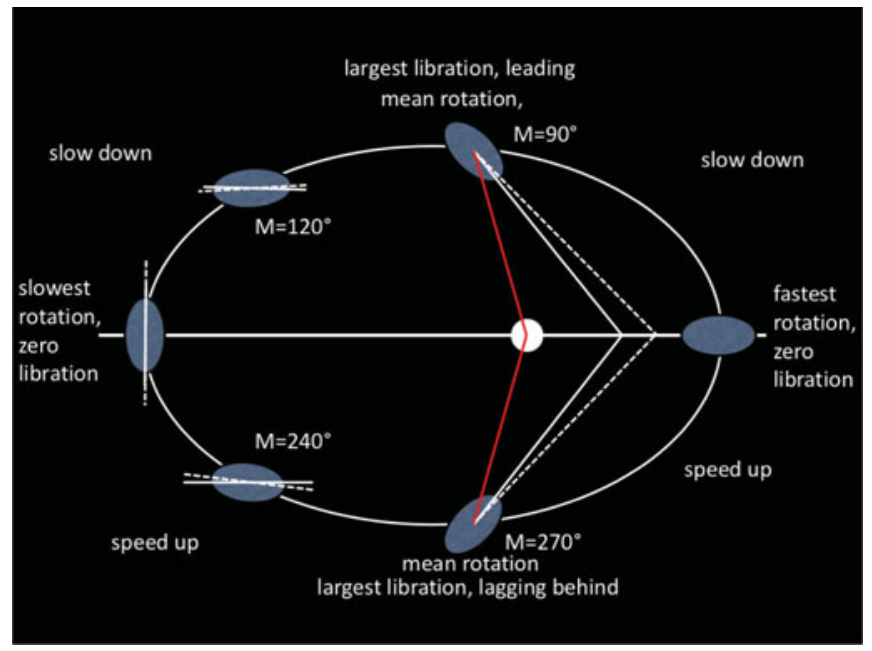

Figure 1. Schematic of the phase of the annual libration of Mercury. White continuous lines represent the approximate orientation of the long axis without libration, dashed lines the orientation including libration.

obtain the libration equation

$$
\frac{d^{2} \gamma}{d M^{2}}+\frac{3}{4} \frac{B-A}{C} e\left(7-\frac{123}{8} e^{2}\right) \sin 2 \gamma=-\frac{3}{2} \frac{B-A}{C}\left(1-11 e^{2}+\frac{959}{48} e^{4}+\cdots\right) \sin M
$$

(Peale 2005). Here harmonic forcing terms of the diurnal frequencies and time-dependent torque components proportional to the small libration angle have been neglected. This equation has the form of a forced harmonic oscillator and the libration will oscillate with a period equal to the orbital period.

The phase of the libration is illustrated in Fig. 1. For mean anomalies between 0 and 180 degrees, the long axis of Mercury is ahead of the direction to the sun in the sense of the rotation of Mercury. Therefore, the gravitational torque exerted by the sun on Mercury will tend to decelerate the rotation. For mean anomalies larger than 180 degrees, the torque tends to accelerate the rotation. The rotation speed is thus largest at perihelion and smallest at aphelion. As for a pendulum passing through its equilibrium position at highest velocity, Mercury passes through the equilibrium of zero libration at the perihelion and its long axis there is in the direction to the sun. Although the rotation slows down for mean anomalies increasing from 0 to 90 degrees, the libration increases as the rotation is faster than average. At a mean anomaly of 90 degrees, Mercury reaches its largest libration leading with respect to a constant mean rotation and rotates at the mean rotation rate. Since Mercury's rotation continues to slow down when further advancing in its orbit, the libration decreases but remains positive until it becomes zero at aphelion. In the second half of its orbit, the long axis of Mercury lags behind the orientation for constant rotation until it again reaches zero libration at the perihelion.

The libration amplitude can immediately be obtained from libration Eq. (2.4), but we first discuss the case of a liquid core. When the core is in addition assumed to be spherically symmetric and not interacting with the mantle, the total torque of the sun is exerted on the solid silicate shell and only the shell with polar moment of inertia $C^{m}$ 
librates with amplitude $g_{M}$ given by

$$
g_{M}=\frac{3}{2} \frac{B-A}{C^{m}}\left(1-11 e^{2}+\frac{959}{48} e^{4}+\cdots\right) .
$$

The libration is linearly dependent on the equatorial moment of inertia difference $B-A$, which is related to the torque, and is inversely proportional to the shell's polar moment of inertia, which represents the rotational inertia (resistance to rotational forcing). When the core is solid, both the silicate shell and core librate with an amplitude that is given by the same equation in which the polar moment of the shell must be replaced by the total polar moment of inertia of Mercury. This difference is key to the interpretation of the libration. For a solid core, the expected libration amplitude is about 190 $\mathrm{m}$ (the polar moment of inertia of Mercury is determined from the same radar observations which also yield an accurate estimate of the obliquity, Margot et al. 2012), less than half the observed value. This demonstrates that Mercury's core must be (at least partially) liquid. Since all other quantities on which the libration amplitude depends are sufficiently well known, libration is thus seen to provide an accurate estimate of the polar moment of inertia of the mantle. This, in turn, can be used to estimate the radius of the core.

Before describing results about the interior structure, we first need to assess the importance of up to now neglected effects on the libration. First, Mercury's core interacts with the mantle in different ways. However, Peale et al. (2002) and Rambaux et al. (2007) (see also Van Hoolst 2007) have shown that all direct interactions between the liquid core and the mantle, such as viscous coupling, electromagnetic coupling, gravitational coupling and pressure coupling related to topography of the core-mantle boundary, have a negligibly small effect on the libration amplitude that is well below the current measurement precision.

An inner core interacts directly with the mantle through gravitational coupling if the long axes of inner core and mantle are not aligned. The global magnetic field observed by Mariner 10 and MESSENGER, if caused by dynamo action, suggests that Mercury contains a growing solid inner core in a liquid outer core. The size of the inner core is currently not constrained but it cannot be excluded that it might possibly even be relatively large although its size is limited by the global radial contraction of about $7 \mathrm{~km}$ estimated from Mercury's tectonics (Byrne et al. 2014). For small inner cores, the effect of an inner core on Mercury's 88 days libration amplitude is below the observational precision. If Mercury's inner core is larger than at least $1000 \mathrm{~km}$ it could, however, be of the order of the current observational uncertainty and be larger than the future BepiColombo observational precision expected to be about $10 \mathrm{~m}$ or somewhat smaller (Pfyffer et al., 2011) (see e.g. Fig. 2, Van Hoolst et al. 2012, Dumberry et al. 2013). Gravitational coupling between the inner core and the mantle slightly reduces the mantle libration by up to $20 \mathrm{~m}$ and therefore the mantle moment of inertia derived from the libration is also somewhat smaller than if derived from Eq. (2.5) (Van Hoolst et al. 2012).

The observed libration amplitude and obliquity, together with the recent determination of the gravity field of Mercury by MESSENGER provide strong constraints on the radial mass distribution in Mercury. Libration stands out because it provides an estimate of a subsystem of the planet (the silicate part) and not, as for the other data, of the whole planet. The geodesy data set strong limits on the size of the core in particular. According to Hauck et al. (2013) and Rivoldini \& Van Hoolst (2013) the core radius is about $2000 \mathrm{~km}$ with an uncertainty of only about $2 \%$. 


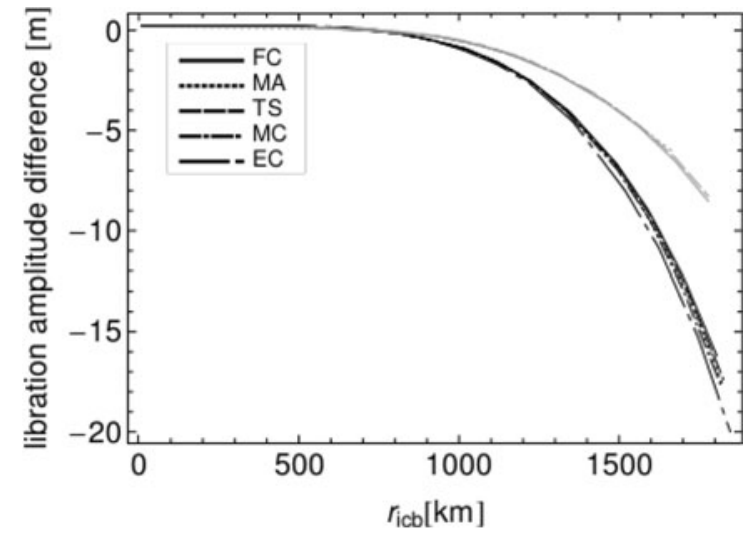

Figure 2. Difference in libration amplitude (in $\mathrm{m}$ ) due to the inner core as a function of inner core radius for different models of the interior structure of Mercury (Van Hoolst et al. 2012)

\section{Large icy satellites}

Several lines of evidence strongly indicate that the largest icy satellites of the solar system (Ganymede, Titan, Callisto, and Europa) all have a subsurface ocean beneath their surface (Khurana et al., 1998, Kivelson et al., 2002, Béghin et al. 2010, Baland et al., 2012, Iess et al. 2012). Essential properties of the subsurface oceans and overlying ice shell, including their size, are not well known. Further insight into the ocean and ice shell characteristics can be obtained from several methods, including those already used for the ocean detection: magnetic observations, observations of the satellite tides (e.g. Wahr et al., 2006) and rotation (e.g. Baland et al., 2011). In particular, the forced diurnal librations, which have not yet been unambiguously detected so far for large icy satellites, might provide useful constraints.

By using an angular momentum approach as for Mercury, the diurnal libration amplitude $g_{s}$ of an entirely solid and rigid satellite is seen to depend on the moments of inertia of the satellite and on the orbital eccentricity:

$$
g_{s}=6 \frac{B-A}{C} e .
$$

Of the four largest icy satellites, Europa has the largest rigid libration amplitude of about $134 \mathrm{~m}$. Titan's amplitude is about $50 \mathrm{~m}$, and the libration amplitude of Callisto and Ganymede is about $12 \mathrm{~m}$ and $10 \mathrm{~m}$, respectively (Van Hoolst et al. (2013), see also Comstock \& Bills (2003)).

The phase of the libration is illustrated in Fig. (3) and can be explained as follows. The direction of the long axis is approximately into the direction of the empty focus (Murray and Dermott 2000). Therefore, the long axis of the satellite is lagging behind the direction to the planet in the sense of the rotation for mean anomalies between 0 and 180 degrees, and is ahead of that direction for mean anomalies larger than 180 degrees. This is opposite to the case of Mercury. The gravitational torque exerted by the planet on the satellite will tend to speed up the rotation in the first half of the orbit and to slow it down in the second half. The rotation speed is thus smallest at pericenter and largest at apocenter. The largest libration will be reached at a mean anomaly of 270 degrees when the rotation is decelerating and reaches the mean rotation. The libration is smallest and lagging the rotation at a mean anomaly of 90 degrees when the rotation is accelerating and reaches the mean rotation rate. 


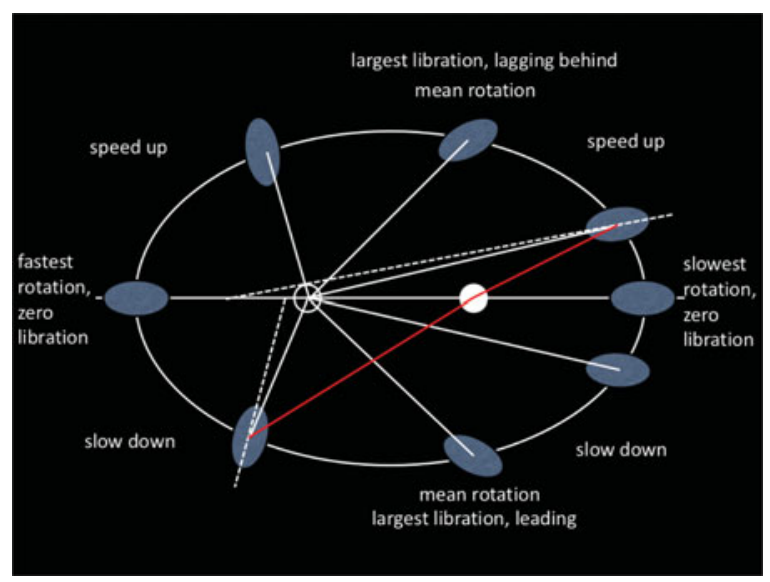

Figure 3. Schematic of the phase of the diurnal libration of synchronous satellites. White continuous lines represent the approximate orientation of the long axis without libration, dashed lines the orientation including libration.

Since the subsurface ocean is close to the surface, one might expect that the real libration amplitude would be much larger if the shell could be considered to be librating almost independently of the deeper interior (Van Hoolst et al. 2008). However, the thin ice shell responds to the gravitational forcing of the central planets not only by libration but also by deformation (tides). As pointed out by Goldreich and Mitchell (2010) this strongly limits the libration amplitude. This reduction can be understood by considering the limit case of a satellite with an ocean that reaches up to the surface. The gravitational and, to a lesser extent, the geometric shape of the satellites is strongly determined by the static tides and the centrifugal acceleration which result in synchronously rotating satellites with a triaxial ellipsoidal shape. On top of these static tides are dynamic tides, mainly with an orbital period. If the ocean extends to the surface (or better if the satellite is entirely liquid) the total tidal bulge (static + periodic) would be in the direction to the central planet at any moment. In that situation, the central planet cannot exert a gravitational torque on the satellite and there would be no libration.

The libration of an icy satellite with a liquid layer can be studied by considering the change in angular momentum of each solid and liquid layer. The angular momentum of a layer changes due to the gravitational torque of the planet on the static shape and on the periodic tidal bulges but also by interaction torques between the different layers. Viscous and electromagnetic torques can probably be neglected because the spin up times associated with these torques are expected to be many orders of magnitude longer than the libration period as in the case of Mercury. The torques to be considered then are the gravitational torques between the static and tidal bulges of the different layers and the pressures torques of the liquid layers on the surrounding solid layers induced by the changing gravitational field resulting from orientation changes and periodic tidal bulges of the different layers. Figures 4 and 5 illustrate the possible different orientation and periodic tidal bulges of the layers resulting in interlayer torques for a satellite (likely representative of Ganymede) with two liquid layers - a subsurface ocean and a liquid iron outer core - and three solid layers - the outer ice shell, a mantle, and a solid iron inner core.

Periodic zonal tides are a separate class of tides to be considered since they change the polar moment of inertia and therefore the rotational response of the layers to forcing. The polar moment of inertia is largest at pericenter and smallest at apocenter. It therefore decreases in the first half of the orbit, effectively contributing to the acceleration of the 


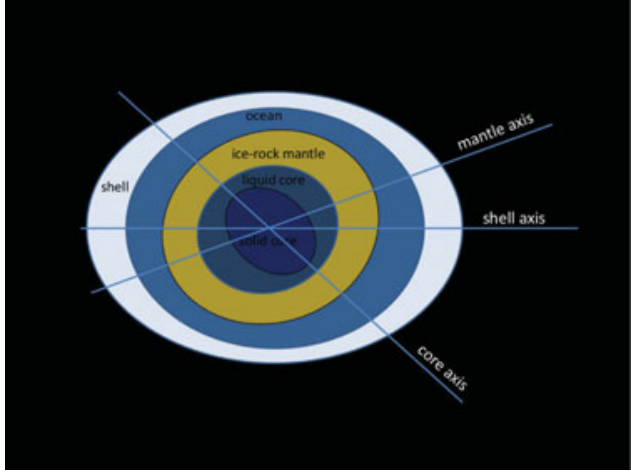

Figure 4. Equatorial cross section of the static shape of an large icy satellite

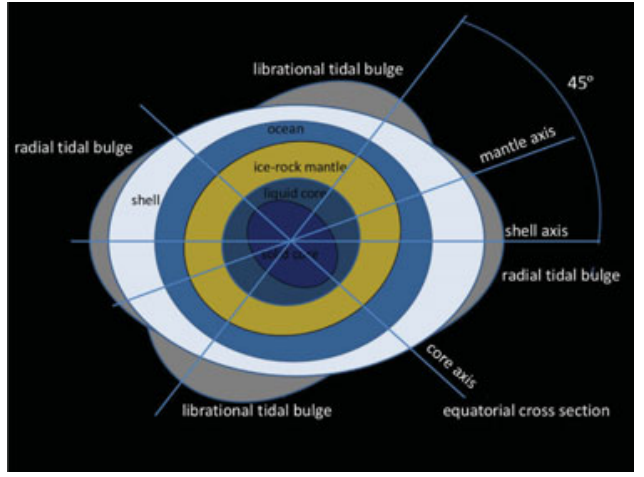

Figure 5. Orientation of the periodic radial and librational tidal bulge of the ice shell

rotation, and increases in the second half, contributing to the deceleration. As a result, the zonal tides increase the libration.

The libration amplitude of the ice shell above an ocean is inversely proportional to the shell moment of inertia because it represents the rotational inertia and proportional to a forcing term which consists of three contributions: the external gravitational and associated pressure forcing of the shell, the forcing of the shell due to coupling between the librational bulges and the static bulges of different layers, and the effect of the zonal tides (Van Hoolst et al. 2013). The last term is at most $10 \%$ of the external torque due to the planet, and the first two terms oppose each other. Interaction between the periodic and static bulges of different layers, in particular between the static bulge of the solid interior and the librational bulge of the ice shell (see Fig. 5), can periodically force the shell libration, in contrast to the torque between static tidal bulges, which cannot force libration and is zero when the libration angles are zero. This interlayer forcing of the libration opposes the forcing due to the central planet as can be seen from the geometry of the bulges, with the librational bulge approximately 45 degrees ahead of the static bulges (see Fig. 5).

The libration amplitude is rather insensitive to the thickness of the ice shell, as was suggested by Goldreich and Mitchell (2010), and is close to the value of the libration for a satellite without subsurface ocean. It mainly depends on the rigidity and the viscosity of the ice shell (see also Jara-Orué and Vermeersen 2014). Figs. 6 and 7 show the dependence on rigidity and viscosity for a model of the interior structure of Ganymede with a subsurface ocean at a depth of $50 \mathrm{~km}$ below the surface. If the ocean is assumed to be freezed out and the whole interior were to be rigid, the libration amplitude would be $8.86 \mathrm{~m}$ for the particular model considered. With an ocean and considering elastic behavior, the libration amplitude increases almost linearly with increasing rigidity of the ice shell by about $2 \mathrm{~m} / \mathrm{GPa}$ (Fig. 6). For viscosities of the ice shell below about $10^{15} \mathrm{~Pa} \mathrm{~s}$ (for an ice shell rigidity of $3.3 \times 10^{9} \mathrm{~Pa}$ ), the libration amplitude decreases to about $1 \mathrm{~m}$. The Maxwell timescale for a viscosity of $10^{15} \mathrm{~Pa}$ s and an ice shell rigidity of $3.3 \times 10^{9} \mathrm{~Pa}$ is close to the orbital period of Ganymede. Therefore, the behavior will be be essentially elastic for larger viscosities and tend to that of a viscous fluid for lower viscosities.

\section{Acknowledgements}

I am grateful to my collaborators on these topics: Rose-Marie Baland, Attilio Rivoldini, and Antony Trinh. 


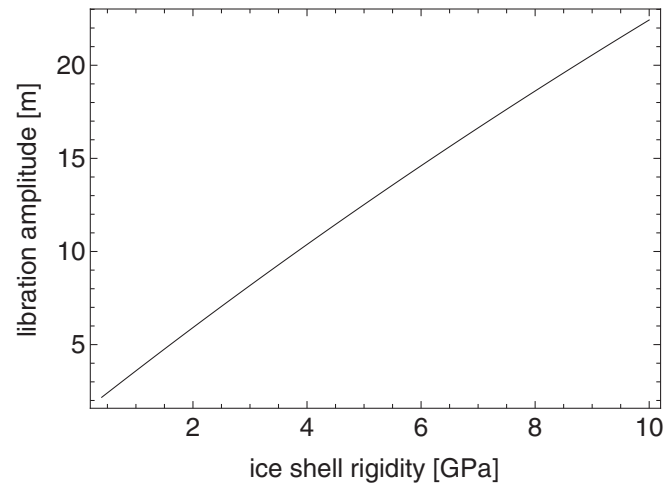

Figure 6. Libration amplitude of Ganymede as a function of the rigidity of the ice shell

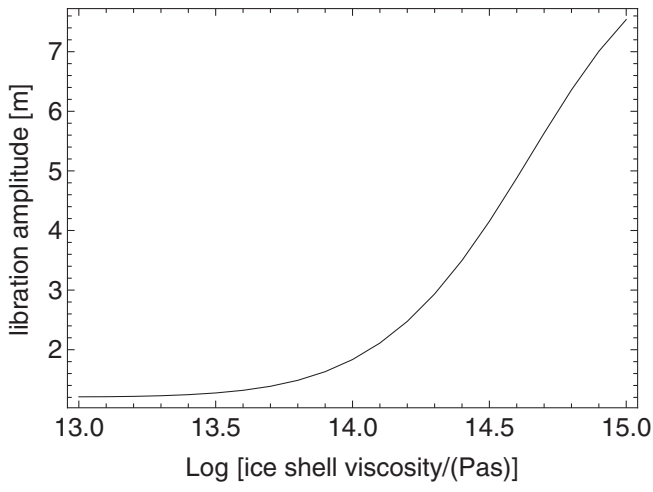

Figure 7. Libration amplitude of Ganymede as a function of the ice viscosity of the shell

\section{References}

Baland, R.-M., Van Hoolst, T., Yseboodt, M., \& Karatekin, O., 2011, A\&A, 530, A141

Béghin, C., Sotin, C., \& Hamelin, M., 2010, C. R. Geoscience, 342, 425

Byrne, P. K., Klimczak, C., Celal Sengor, C. A., Solomon, S. C., Watters, T. R., \& Hauck II, S. A., 2014, Nature Geoscience, 7, 301

Comstock, R. L. \& Bills, B. G. 2003, J. Geophys. Res., 108(E9), 5100

Dumberry, M., Rivoldini, A., Van Hoolst, T., \& Yseboodt, M., 2010, Icarus, 225, 62

Goldreich, P. M. \& Mitchell, J. L. 2010, Icarus, 209, 631

Hauck, S. A., Margot, J.-L., Solomon, S. C., Phillips, R. J., Johnson, C. L., Lemoine, F. G., Mazarico, E., McCoy, T. J., Padovan, S., Peale, S. J., Perry, M. E., Smith, D. E., \& Zuber, M. T., 2013. J. Geophys. Res., 118, 1

Iess, L., R. A. Jacobson, M. Ducci, D. J. Stevenson, J. I. Lunine, J. W. Armstrong, S. W. Asmar, P. Racioppa, N. J. Rappaport, P. Tortora, 2012, Science, 337, 457

Jara-Orué, H. \& Vermeersen, B. L. A. 2014, Icarus, 229, 31

Khurana, K. K., Kivelson, M. G., Stevenson, D. J., Schubert, G., Russell, C. T., Walker, R. J., \& Polanskey, C. 1998, Nature, 395, 777

Kivelson, M. G., Khurana, K. K., \& Volwerk, M., 2002, Icarus, 157, 507

Margot, J.-L., Peale, S. J., Jurgens, R. F.,. Slade, M. A., \& Holin, I. V., 2007, Science 316, 710

Margot, J.-L., Peale, S. J., Solomon, S. C., Hauck, S. A., Ghigo, F. D., Jurgens, R. F., Yseboodt, M., Giorgini, J. D., Padovan, S., \& Campbell, D. B., 2012, J. Geophys. Res., 117, E00L09

Murray, C. D. \& Dermott, S. F., 2000, Solar System Dynamics, Cambridge University Press

Peale, S. J., 1976, Nature 262, 765

Peale, S. J., 2005, Icarus, 178, 4

Peale, S. J., Phillips, R. J., Solomon, S. C., Smith, D. E., \& Zuber, M. T., 2002, Meteoritics and Planetary Science, 37, 1269

Pettengill, G. H. \& Dyce, R. B., 1965, Nature, 206, 1240

Pfyffer, G., Van Hoolst, T., \& Dehant, V., 2011, Planetary and Space Science, 59, 848

Rambaux, N., Van Hoolst, T., Dehant, V., \& Bois, E., 2007, A\&A, 468, 711

Rivoldini, A., \& Van Hoolst, T., 2013, Earth and Planetary Science Letters, 377-378, 62

Smith, D. E., \& 16 co-authors, 2012, Science, 336, 214-217

Van Hoolst, T., 2007, Treatise on Geophysics, ed. G. Schubert, Vol.10: Planets and Moons (ed. T. Spohn), 123

Van Hoolst, T., Rambaux, N., Karatekin, Ö., Dehant, V., \& Rivoldini, A. 2008, Icarus, 195, 386

Van Hoolst, T., Rivoldini, A., Baland, R.-M., \& Yseboodt, M., 2012, Earth and Planetary Science Letters, 333-334, 83

Van Hoolst, T., Baland, R.-M., \& Trinh, A. 2013, Icarus, 226, 299

Wahr, J. M., Zuber, M. T., Smith, D. E., \& Lunine, J. I., 2006, J. Geophys. Res., 111, E12005 